\title{
Efectividad de la gestión de costos en las PYMEs agroindustriales de la Región Tacna, 2015
}

\author{
Effectiveness of cost management in agroindustrial SMEs of the \\ Tacna Region in 2015
}

\author{
${ }^{1}$ Violeta Quevedo Porras
}

\begin{abstract}
RESUMEN
Las empresas de hoy se desenvuelven en entornos muy cambiantes y de alta competencia. Siendo, en los últimos años, la contabilidad de costos la que ha experimentado una mayor cantidad de cambios; provocando una gran necesidad de conocer cómo planificar, organizar, ejecutar y controlar dentro de las empresas, esto con el objeto de mejorar su estructura de costos y tomar decisiones acordes con las necesidades actuales. En este contexto, resultó fundamental determinar cuál es el nivel de efectividad de la gestión de costos en las PYMEs agroindustriales de la Región Tacna. La población que se consideró para efectuar el presente trabajo de investigación corresponde al personal directivo de las PYMEs agroindustriales ubicadas en la Región Tacna, las cuales están inscritas en la Dirección Regional de la Producción del Gobierno Regional de Tacna, esta investigación se realizó en 21 PYMEs agroindustriales. El tipo de investigación fue exploratoria y el diseño, descriptivo. Se utilizó la encuesta como instrumento utilizando la escala de Likert. Los resultados indicaron que la gestión de costos en las PYMEs agroindustriales de la Región Tacna es efectiva.
\end{abstract}

Palabras clave: Costos, efectividad, gestión de costos.

\begin{abstract}
The companies of today act in a very changing environment and of high competition. In addition, in recent years cost accounting has experienced a greater number of changes causing a great need to know how to plan, organize, manage and control the firms. This with the objective of improve cost structures and make decisions according to current needs. In this context, it is essential to determine what is the level of cost management effectiveness in the agro-industrial SMEs of the Tacna Region. The population is formed by the managerial staff of the agro-industrial SMEs located in the Tacna Region, which are registered in the Regional Direction of Production of the Regional Government of Tacna. This research was carried out in 21 agro-industrial SMEs. The type of research was exploratory and the design, descriptive. A survey was used as an instrument using the Likert scale. The results indicated that cost management in the agro-industrial SMEs of the Tacna Region is effective.
\end{abstract}

Key words: Costs, effectiveness, cost management. 


\section{INTRODUCCIÓN}

Cada día las empresas impulsan nuevos enfoques en su dirección y organización, debido a la compleja globalización y el entorno competitivo en el que se desenvuelven. Se puede afirmar que, las empresas de hoy se desenvuelven en entornos muy cambiantes, siendo fundamental la gerencia del cambio, la que traerá beneficios efectivos si el equipo está comprometido con ello; si sus miembros están alineados a una misma estrategia, a la misión y visión de la empresa; pero más aún, permitirá aumentar la productividad y reducir costos con la gestión de costos, útil para la toma de decisiones.

En ese sentido, el presente estudio de investigación plantea un modelo de gestión de costos con el fin de determinar cuál es el nivel de efectividad de costos en las PYMEs agroindustriales de la Región Tacna. En otras palabras, si la empresa cuenta con una adecuada planificación, organización, ejecución y control de los costos, entonces, la empresa podrá concretar sus objetivos satisfactoriamente.

\section{PLANTEAMIENTO DEL PROBLEMA}

\section{Descripción del problema}

Las necesidades de información relevante en las PYMEs han ido evolucionando tan rápido, que conforme van cambiando las condiciones de mercado y la sociedad misma, es necesario conocer mejor sus estructuras de costos. Asimismo, se observa que la carencia de una gestión en los costos no permite un adecuado conocimiento, así como emplear información fehaciente sobre los costos para tomar mejores decisiones.

De la misma manera, esta carencia imposibilita maximizar el rendimiento del negocio, siendo cada vez más notoria una deficiencia al momento de planificar, organizar, ejecutar y controlar.

\section{Antecedentes del problema}

Rincón y Villarreal (2010), en su libro Costos, decisiones empresariales, afirman que los autores de las grandes empresas multinacionales para mejorar la comercialización y competitividad invierten en el conocimiento de su empresa, es decir, creando o implementando sistemas de información de costos con mayor exactitud. Generalmente, las pequeñas y medianas empresas fabrican sin conocer los datos de sus sistemas productivos, sin una información financiera y sin un sistema contable de costos y presupuestos, haciéndolos débiles en los procesos de negociación y competitividad por falta de conocimientos.

Según Udolkin (2015), en su libro Contabilidad de costos y de gestión, las empresas se desenvuelven en un entorno muy cambiante y de alta competencia, con clientes más informados y exigentes, donde los productos y los canales de distribución son más complejos, con sistemas de producción más automatizados y con productos con ciclos de vida más cortos.

En este contexto, las empresas requieren tener sistemas de información contable que provean información más detallada y precisa de las operaciones de la empresa de la que usualmente proveen los sistemas de costeo tradicionales.

\section{Problemática de la investigación}

La agroindustria de las PYMEs en la Región Tacna está constituida por la elaboración de una gran variedad de productos, cuya materia prima es diversa, aunque se destaca ampliamente el olivo y la vid. Las PYMEs agroindustriales de la Región Tacna fabrican los siguientes productos: aceite de oliva, licores afrutados, vino y pisco de diferentes tipos y calidades, bebidas, productos lácteos (quesos, yogurt), entre otros. Además, si se compara el rendimiento actual de los cultivos por hectárea con los últimos 10 años, se evidencia un incremento significativo que nos coloca en una situación potencial para ingresar a una agroindustria más competitiva; y sobre todo, para la exportación, ya que el mercado internacional está demandando productos de zonas poco contaminadas.

Tacna destaca por ser una de las principales regiones productoras de pisco de mayor calidad 
en el país; por ende, el crecimiento de las exportaciones no solo ha sido en volumen, sino también en precio. Esta situación ha implicado mejoras en la calidad de este producto y acceso a mercados más exigentes.

Entonces, esto nos lleva a pensar si los sistemas, métodos y técnicas usados en el costeo de productos que se están implementando, reflejan un mejor control de los costos, es decir: ¿Realmente estamos proporcionando la información correcta sobre el margen y rentabilidad de los productos y servicios? ¿Cuál es el nivel de efectividad de la gestión de costos en las PYMEs agroindustriales?

Ante este escenario, es imprescindible llevar a cabo un modelo de gestión idóneo para que las PYMEs agroindustriales puedan decidir sobre la planificación, el control y la dirección, con la finalidad de mejorar. A partir de las últimas décadas del siglo XX, las empresas están experimentando un proceso de cambios revolucionarios, pasando de una situación de protección regulada a entornos abiertos altamente competitivos.

Esta situación de transformaciones constantes del ambiente de negocio es vital para que las PYMEs agroindustriales puedan mantener e incrementar su participación en el mercado; $y$ al mismo tiempo, saber cómo analizar y evaluar los procesos de su negocio, es decir, conocer detalladamente el sistema de medición de desempeño para alcanzar el nivel de competencia requerido.

Dicho impacto del entorno competitivo es una de las principales causas del fracaso de las PYMEs. Este entorno cambiante y la competitividad son factores de exigencia para que las PYMEs puedan mejorar en distintos aspectos, a partir de una correcta gestión de costos y mediante soluciones objetivamente válidas. Además, se debe considerar el hecho de ser consecuente con las decisiones, ya que en frecuentes ocasiones terminan siendo la causa de la desaparición de la empresa.

Asimismo, la falta de conocimientos provocará una deficiente planeación y desorden en sus respectivos procesos; y así las empresas derrocharán poco a poco su presupuesto. Por lo tanto, para mantenerse y crecer en un modelo de globalización, deben educarse, aliarse y organizarse.

\section{Formulación del Problema}

\section{Problema general}

¿Cuál es el nivel de efectividad de la gestión de costos en las PYMEs agroindustriales de la Región Tacna, 2015?

\section{Problemas específicos}

a. ¿Cuál es el nivel de efectividad de la planificación de costos en las PYMEs agroindustriales de la Región Tacna, 2015?

b. ¿Cuál es el nivel de efectividad de la organización y ejecución de costos en las PYMEs agroindustriales de la Región Tacna, 2015?

c. ¿Cuál es el sistema de costos más empleado en las PYMEs agroindustriales de la Región Tacna, 2015?

d. ¿Cuál es el nivel de efectividad del control de costos en las PYMEs agroindustriales de la Región Tacna, 2015?

e. ¿Cuál es la efectividad en las PYMEs agroindustriales de la Región Tacna, 2015?

\section{Justificación e importancia}

"Aquellas empresas que no se aboquen a realizar cambios drásticos para manejar sus costos no van a lograr sobrevivir". (Cuervo y Osorio, 2011, p. 249).

El sector manufacturero en Perú, que tiene el mayor peso en el cálculo del Producto Bruto Interno (PBI), presenta la menor previsión de crecimiento para el año 2019, con "crecimiento nulo" de acuerdo al Banco Central de Reserva del Perú.

De esta forma, se reconoce la importancia económica del crecimiento del sector 
agroindustrial en Tacna, debido a su ubicación estratégica, la cual es limítrofe con dos países. Por ello, existe un gran potencial para dinamizar sus exportaciones y asumir el reto de prepararse para competir y responder a las nuevas oportunidades que ofrece el mercado externo.

Por otro lado, la globalización, la escasez de recursos y la fuerte competencia exigen a las empresas diversificación, consolidación e implementación de un sistema que les permita adaptarse al entorno y conseguir soluciones eficaces para los nuevos problemas que deben enfrentar. Inclusive, cuando gran parte de nuestra producción es comercializada a intermediarios o llevada por empresarios a otros países, donde la reclasifican y exportan como producto extranjero hacia mercados más competitivos.

Las PYMEs agroindustriales no escapan de esta realidad, ya que este sector se ha visto afectado por la legislación gubernamental y la regulación de importaciones de los insumos básicos requeridos, lo que ha ocasionado un incremento en sus costos de producción.

Ello requiere conocer con precisión los elementos de la gestión de costos para llevar a cabo una adecuada planificación, organización, ejecución y control; lo que permitirá obtener información fidedigna sobre los costos; y así se determinará la rentabilidad de los productos, por ende, hasta donde se pueden bajar los precios de venta para enfrentar a la competencia sin obtener pérdidas y evitar que se tomen decisiones basadas en la intuición o la experiencia, y así poder ampliar la gama de productos competitivos y posicionados exitosamente en el mercado internacional.

En Tacna se encuentran 27 PYMEs, de acuerdo al padrón de PYMEs agroindustriales proporcionado por la Dirección Regional de la Producción del Gobierno Regional de Tacna, y se espera que sean más las PYMEs agroindustriales las que proporcionen estabilidad y viabilidad a una gran cantidad de pequeños, medianos y grandes agricultores, tanto desde el punto de vista económico como desde el punto de vista cultural, ya que servirá de nexo para mejorar la calidad y competitividad en la gestión agrícola, requisitos indispensables para abordar los mercados internacionales.

Sin embargo, existe el caso de la empresa Conaisa, que de acuerdo al último Informe Económico y Social (2013) del Banco Central de Reserva del Perú (BCR), esta empresa destacó en la comercialización de la agroindustria en diferentes zonas del Perú, principalmente en la exportación. Asimismo, destacó en el mercado nacional, aunque lamentablemente hoy en día esta empresa se encuentra en baja de oficio. Esto implica que las PYMEs agroindustriales de la Región Tacna estén dispuestas a adaptarse a los cambios y condiciones que el mercado y la sociedad actuales exigen.

Hay que considerar que no hay estudios o investigaciones que revelen el grado en que influye la gestión de costos en la efectividad de las PYMEs agroindustriales de la Región Tacna. Por otra parte, es fundamental para la realización de futuras investigaciones, ya que brinda información relevante y coherente sobre el manejo de los costos en las PYMEs del sector agroindustrial, permitiendo acercarse al sector y a su funcionamiento empresarial.

De esta manera, un buen modelo de gestión de costos ayudará a identificar las verdaderas causas por las cuales la empresa incurre en mayores costos. Asimismo, la empresa tendrá información precisa sobre los costos de sus productos; estarán mejor preparadas para asumir los cambios y enfrentar la competencia de mejor manera.

Por lo tanto, buscamos una apropiada implementación de la gestión de costos para evitar decisiones equivocadas, en la medida que proporciona información confiable, objetiva y oportuna para la dirección, con el propósito de percibir los costos como una herramienta para el análisis de la utilidad y toma de decisiones empresariales. 


\section{Objetivos}

\section{Objetivo General}

Determinar el nivel de efectividad de la gestión de costos en las PYMEs agroindustriales de la Región Tacna, 2015.

\section{Objetivos específicos}

a. Determinar el nivel de efectividad de la planificación de costos en las PYMEs agroindustriales de la Región Tacna, 2015.

b. Determinar el nivel de efectividad de la organización y ejecución de costos en las PYMEs agroindustriales de la Región Tacna, 2015.

c. Establecer el sistema de costos más empleado en las PYMEs agroindustriales de la Región Tacna, 2015.

d. Determinar el nivel de efectividad del control de costos en las PYMEs agroindustriales de la Región Tacna, 2015.

e. Analizar la efectividad en las PYMEs agroindustriales de la Región Tacna, 2015.

\section{Hipótesis de la investigación}

\section{Hipótesis general}

La efectividad de la gestión de costos en las PYMEs agroindustriales de la Región Tacna, 2015 es alta.

\section{Hipótesis específicas}

a. La efectividad de la planificación de costos en las PYMEs agroindustriales de la Región Tacna es alta.

b. La efectividad de la organización y ejecución en las PYMEs agroindustriales de la Región Tacna es alta.

c. El sistema de costos por procesos es el más empleado en las PYMEs agroindustriales de la Región Tacna.

d. La efectividad del control de costos en las PYMEs agroindustriales de la Región Tacna es alta.

e. La efectividad en las PYMEs agroindustriales de la Región Tacna es alta.

\section{MARCOTEÓRICO}

Según Guerra (1992), la administración de empresas agropecuarias puede asumirse como un proceso de toma de decisiones (...), un factor determinante del éxito o fracaso de cualquier negocio donde deben desarrollarse como funciones básicas de administración la planificación, organización, ejecución y control. Todo esto basado en los lineamientos dados por Boehlje y Eidman, como se observa en la Figura 1.

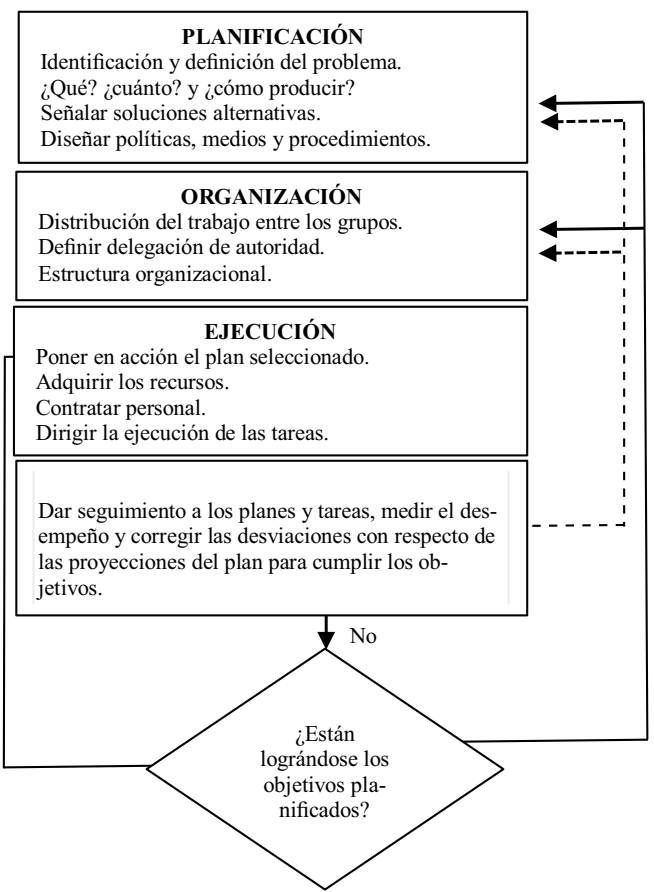

Figura 1. Gestión de costos.

Fuente: Guerra, G.E (1992). Manual de administración de empresas agropecuarias. San José, Costa Rica: Instituto Interamericano de Cooperación para la Agricultura(IICA).

\section{Planificación}

"La planificación consiste en la formulación de un futuro deseado para una organización en el corto, mediano y largo plazo" (Mendieta, 1996, p. 39).

Según Guerra (1992), la planificación podría definirse como la selección de acciones más 
apropiadas para producir los resultados que se desean. Asimismo, es una metodología para decidir correctamente, ya que la decisión envuelve una selección entre dos o más alternativas. En tal sentido, se podría agregar que la planificación es una metodología para

la selección de alternativas.

Según Mendieta (1996), la planificación es un proceso continuo en la medida en que la información disponible dentro o fuera del sistema permita identificar nuevos problemas. La información nueva que se obtiene de la función de control proporciona una retroalimentación de la planificación, razón por la cual se convierte en una etapa importante del sistema total.

Por lo tanto, todo plan estructurado persigue objetivos determinados. Por ello, la esencia de un plan son sus objetivos como empresa, de lograr la efectividad en la gestión de costos. De tal forma, esto permita tomar decisiones acordes con sus necesidades actuales.

De esta manera, aquellos procesos requeridos para definir el nivel de la planificación de costos son:

a. La empresa cuenta con un plan de negocios, el cual se ha desarrollado y se hace el seguimiento constante de su ejecución y el logro de los objetivos/metas establecidos.

b. Se cuenta con un plan de producción debidamente detallado y cuya estructura de costos ha sido debidamente coordinada entre todas las áreas de la empresa.

c. En la empresa se usa datos e información tanto internas como externas para el desarrollo de modelos de planificación y replanificación.

d. Se emplea modelos para analizar y medir unidades disminuidas, dañadas, defectuosas, desechos y desperdicios.

e. La empresa determinó formalmente qué sistema de costos implementar al inicio de sus operaciones y evalúa su efectividad periódicamente, cambiándolo si fuera necesario.

\section{Organización}

"Sheldon (s.f.) menciona que la organización es el proceso de combinar el trabajo del personal de una empresa con los elementos necesarios para su ejecución; de tal manera que las labores que así se ejecuten, sean los mejores medios para la aplicación eficiente, sistemática, positiva y coordinada de los esfuerzos disponibles". (Mendieta, 1996, p.91).

El concepto de organización ha sido definido o empleado de varias maneras por diferentes autores. Sin embargo, se debe considerar antes que la palabra organización se ha usado para denotar: El proceso de agrupar y arreglar diversas partes mutuamente dependientes con el propósito de formar un todo; una unidad formada por varios componentes, los cuales dependen recíprocamente entre sí, aunque cada uno con una función específica; un grupo de personas agrupados para un fin determinado; la estructura ejecutora de una empresa; el personal administrativo de una empresa y los elementos del costo de producción.

Para determinar el grado de organización y ejecución se tiene los siguientes indicadores:

a. Se emplean formatos estandarizados para el requerimiento y compra de insumos, materias primas, materiales auxiliares, productos y mercancías.

b. Se tienen implementados mecanismos de control de los inventarios de materias primas, productos en proceso y productos terminados.

c. Existe un sistema o procedimiento formal de registro, control y remuneración de la mano de obra.

d. Existe un programa de capacitación de los trabajadores, que se cumple y cuya selección de los beneficiados se hace en base a criterios técnicos o según necesidad.

e. Se utilizan métodos de distribución de los costos indirectos de fabricación de forma directa, por el método escalonado o método recíproco.

f. Las bases para aplicar los costos indirectos de fabricación a la producción son el valor, el tiempo o las unidades producidas.

g. Se aplica NIIF para las PYMES.

\section{Ejecución}

La ejecución es el conjunto de procesos realizados para completar el trabajo definido en el plan para la dirección del 
proyecto a fin de satisfacer las especificaciones del mismo. Este grupo de procesos implica coordinar personas $\mathrm{y}$ recursos (...); así como, integrar y realizar las actividades del proyecto conforme al plan para la dirección del proyecto. Durante la ejecución del proyecto, en función a los resultados obtenidos, se puede requerir una actualización de la planificación.(PMBOK, 2013,p. 55).

La ejecución consiste en llevar a cabo los planes escogidos. Una vez que se completa el proceso de planificación y de organización se debe aplicar el sistema de costo seleccionado como mejor alternativa para el costeo de su producción:

a. Sistema de costos por órdenes (S-CO): Si los costos se determinan principalmente en función a las especificaciones e instrucciones contenidas en las órdenes de pedido de los diversos clientes.

b. Sistema de costeo por procesos (S-CP): Si se tiene una acumulación de costos de producción por centro de costos.

c. Sistema de costos estimados (S-CE): Si los costos se determinan en función a la estimación previa de lo que podría costar un producto o un proceso durante cierto periodo de tiempo, con la mayor aproximación posible, en base al conocimiento o experiencia

d. Sistema de costos estándar (S-CES): Si los costos se determinan en función a costos normales presupuestados de materia prima, mano de obra directa y costos indirectos de fabricación.

e. Costos conjuntos ( $\mathrm{S}-\mathrm{CC}$ ): $\mathrm{Si}$ los costos de un proceso de producción generan diversos productos de manera simultánea

f. Sistema de costeo basado en actividades (SABC): Si los costos se determinan principalmente en función a la determinación de las actividades basado en el uso de los recursos.

\section{Control}

Según Guerra (1992), el control es el proceso de medir los resultados del plan, compararlos con el estándar previamente establecido y efectuar los ajustes correspondientes para lograr los objetivos deseados. Esto último es la esencia del control en la empresa agroindustrial.

Aquellas acciones requeridas para rastrear, revisar y regular el progreso y el desempeño del proyecto, para identificar áreas en las que el plan requiera cambios y para iniciar los cambios correspondientes, forman parte del proceso de control.

Por otro lado, en esta etapa se debe influir en los factores que podrían eludir el control integrado de cambios o la gestión de la configuración, de modo que únicamente se implementen cambios aprobados. "Este monitoreo continuo proporciona al equipo del proyecto conocimiento sobre la salud del proyecto y permite identificar las áreas que requieren más atención”. (PMBOK, 2013, p. 56).

En esta etapa se controla los cambios y recomienda acciones correctivas para anticipar posibles problemas y monitorear las actividades del proyecto comparándolas con lo planificado. Además, se suma el procedimiento de retroalimentación que se emplea para mejorar los planes actuales o futuros.

Para Guerra (1992), esta retroalimentación proporciona un ciclo continuo de planificación, organización, ejecución, seguimiento y registro del proceso para pasar luego a la revisión del plan y al proceso de ejecución. De igual manera, con el aprovechamiento de la nueva información obtenida por medio de la función de control. Un buen sistema de control requiere un sistema seguro de registros y una buena habilidad para usarlos. Por otro lado, carecer de registros detallados es como navegar un barco sin brújula; por lo tanto, no hay forma de saber dónde se está, dónde se va a ir ni cuánto tiempo tomará llegar a la meta.

Para la medición del nivel de control de costos, se describe los siguientes indicadores:

a. La información que se genera en la empresa para la toma de decisiones es oportuna, confiable y/o consistente

b. El control de los saldos de las cuentas del estado de situación financiera y estado de resultados es oportuna, confiable y/o consistente 
c. El nivel gerencial de la empresa considera que la gestión de costos es importante para la organización

d. Las diversas áreas involucradas en el control de costos conocen la información, indicadores y metas de la gestión de control de costos

\section{Efectividad empresarial}

La efectividad en las empresas es la cuantificación del logro de las metas. Además, es muy aceptado que se considere a la efectividad como la medida en que la empresa cumple con los objetivos planificados, optimizando el uso de los recursos con que dispone.

Muchos autores relacionan la efectividad con la búsqueda de la eficacia y eficiencia. Sin embargo, de acuerdo con el diccionario de la Real Academia Española (RAE), la eficacia se define como la "capacidad de lograr el efecto que se desea o se espera"; la eficiencia se refiere a la "capacidad de disponer de alguien o de algo para conseguir un efecto determinado"; mientras que la efectividad la considera como un sinónimo del término eficacia.

Al respecto; Mejía (2013), para la medición de la efectividad describe los siguientes indicadores:

\section{a.Cumplimiento del programa de producción}

Es el grado en que se cumple la planificación y elaboración de los productos durante las fechas previstas. Suele ser influenciado por la disponibilidad de las instalaciones, maquinarias, logística y transporte; así como la eficiencia de los equipos de trabajo. En la auditoría de operaciones se revisa la contabilidad detallada y la divisionaria.

\section{b.Cumplimiento del plan de ventas}

Es el grado en que se cumple con colocar en el mercado la producción realizada en los plazos y condiciones previstas. Suele ser influenciado por la disponibilidad y uso propicio de las instalaciones; así como la gestión de la comercialización y de las ventas. En la auditoría de operaciones se revisa el rubro o cuenta.

\section{c. Aprovechamiento de la capacidad instalada}

Es el grado en que se usa de manera racional las instalaciones del área de producción y la capacidad instalada. Suele ser influenciado por la disponibilidad y mantenimiento de las instalaciones, el transporte y manipuleo de las materias primas e insumos; así como la capacidad de producción del equipamiento con que se dispone. En la auditoría de operaciones se revisan los registros contables y el plan contable.

\section{d.Nivel de inventarios}

Es el grado en que se usa de manera racional el capital invertido en inventarios con relación a las ventas. Suele ser influenciado por la eficiencia en el uso de las materias primas e insumos, los niveles de reposición establecidos, la compra oportuna y el cumplimiento en el pago a los proveedores. En la auditoría de operaciones se revisan los procedimientos contables.

\section{e. Procesos empleados}

Es el grado en que los procesos empleados permiten cumplir con la planificación realizada. Suele ser influenciado por la disponibilidad y mantenimiento de la infraestructura y equipamiento; así como el transporte y la capacidad de las instalaciones. En la auditoría de operaciones se revisa la descripción y dinámica contable.

\section{f.Aceptación de los productos}

Es el grado en que los productos son aceptados como "buenos" por los clientes. Suele ser influenciado por la eficiencia en la gestión de la comercialización y de las ventas, la atención y solución de reclamos y quejas de los clientes; así como la eficiencia en la gestión de calidad de la producción. En la auditoría de operaciones se revisan los estados financieros.

\section{MARCO METODOLÓGICO}

El presente trabajo de investigación es de tipo exploratorio, el análisis y alcance de los resultados es descriptivo. 
La población que se consideró para efectuar la investigación corresponde al personal directivo de las PYMEs agroindustriales ubicadas en la Región Tacna, las cuales están inscritas en la Dirección Regional de la Producción del Gobierno Regional de Tacna, según se muestra en la Tabla 1. Debido a que la población mencionada no tiene un gran tamaño y al existir cierta resistencia en la aplicación de la encuesta, se decidió efectuar un censo y recolectar la información necesaria de toda la población. Es por ello que, la muestra para esta investigación es en 21 PYMEs agroindustriales.

Tabla 1

PYMES agroindustriales de la Región Tacna

\begin{tabular}{|c|c|c|}
\hline $\begin{array}{c}\text { CÓDIGO } \\
\text { CIIU }\end{array}$ & DESCRIPCIÓN & CANTIDAD \\
\hline 15130 & $\begin{array}{l}\text { Elaboración y conservación de } \\
\text { frutas, legumbres y hortalizas. }\end{array}$ & 24 \\
\hline 15142 & $\begin{array}{l}\text { Elaboración de aceites y grasas } \\
\text { de origen vegetal y animal. }\end{array}$ & $\mathrm{s}$ \\
\hline 15202 & Elaboración de productos lácteos. & 1 \\
\hline 15499 & $\begin{array}{l}\text { Elaboración de otros productos } \\
\text { alimenticios. }\end{array}$ & os \\
\hline 1123 & $\begin{array}{l}\text { Cultivo de hortalizas y legumbres; } \\
\text { importación de insumos como la } \\
\text { aceituna; y exportaciones de } \\
\text { productos agrícolas. }\end{array}$ & $\begin{array}{l}\text { s: } \\
\text { la }\end{array}$ \\
\hline 4620 & $\begin{array}{l}\text { Ventas al por mayor de materias } \\
\text { primas agropecuarias y animales } \\
\text { v i v o s; p r o d u c c i ó n, } \\
\text { comercialización y exportación de } \\
\text { productos agroindustriales. }\end{array}$ & $\begin{array}{l}\text { as } \\
\text { es } \\
\text { de }\end{array}$ \\
\hline \multirow[t]{2}{*}{15520} & Elaboración de vinos. & 3 \\
\hline & TOTAL & 35 \\
\hline
\end{tabular}

Fuente: Dirección Regional de la Producción del Gobierno Regional de Tacna

\section{Recolección de datos}

- Tipo de instrumento: Encuesta con escala tipo Likert con las siguientes escalas: Nunca, rara vez, a veces, frecuentemente y siempre.

- Ámbito de aplicación: Dirigido a directivos: Gerente General, Gerente/Jefe de Contabilidad, Gerente/Jefe de Producción o Administrador. Personas que tengan conocimiento de la variable a estudiar en su empresa.
- Método de validación: Validez del instrumento con expertos (0.947) y confiabilidad: 0.927 .

\section{Procesamiento de datos}

El procesamiento de datos se hizo de forma automatizada con la utilización de medios informáticos. Para ello, se emplearon:

- El soporte informático SPSS 22.0: Paquete con recursos para el análisis descriptivo con tablas de doble entrada que permiten ver el comportamiento conjunto de la variable y el cálculo de medidas inferenciales como:

El desarrollo de la prueba T de Student y la elaboración de las tablas de doble entrada

La verificación de la tercera hipótesis específica con el anális de varianza (ANOVAde un factor)

El desarrollo de la R de pearson

- Microsoft Office Excel: Para los recursos gráficos y funciones específicas, los cuales facilitaron el ordenamiento de datos con la elaboración de gráfica circulares y de barras.

\section{DISCUSIÓN DE RESULTADOS}

De acuerdo a los resultados obtenidos en la encuesta, detallada en la Tabla 2, con la prueba estadística T de Student y un nivel de confianza del $95 \%$, se demuestra que la efectividad de la planificación de costos en las PYMEs agroindustriales de la Región Tacna es alta.

Tabla 2

Nivel de planificación de costos en las Pymes agroindustriales de la Región Tacna

\begin{tabular}{|lcc|} 
Nivel & Frecuencia & Porcentaje \\
\hline Bajo & 2 & $10 \%$ \\
Regular & 8 & $38 \%$ \\
Alto & 11 & $52 \%$ \\
Total & 21 & $100 \%$ \\
\hline
\end{tabular}


De acuerdo a la Tabla 3, se verifica con la prueba estadística $\mathrm{T}$ de Student a un nivel de confianza del $95 \%$, que la organización y ejecución en las PYMEs agroindustriales de la Región Tacna es alta.

Tabla 3

Nivel de organización y ejecución en las Pymes agroindustriales de la Región Tacna

\begin{tabular}{|lcc|} 
& Frecuencia & Porcentaje \\
\hline Bajo & 1 & $5 \%$ \\
Regular & 8 & $38 \%$ \\
Alto & 12 & $57 \%$ \\
Total & 21 & $100 \%$
\end{tabular}

Fuente: Elaborado en base a la encuesta

Para nuestra tercera hipótesis se contrasta con Udolkin (2015), quién asegura que el sistema de costeo que utiliza una empresa obedece a las características del proceso de producción de cada producto que elabora o a las características operativas de cada servicio que brinda. Por lo tanto, las PYMEs agroindustriales al comprender la producción, industrialización y comercialización es que se verifica que el sistema de costos por procesos sea el más empleado.

De esta manera con la prueba estadística $\mathrm{T}$ de Student y un nivel de confianza del $95 \%$, se demuestra que el Sistema de costos por procesos no es el más empleado en las PYMEs agroindustriales de la Región Tacna.

Para alcanzar este tercer objetivo específico se consideró pertinente hacer 2 grupos complementarios de preguntas. En el primer grupo, constituido por 6 preguntas se presentaron cada uno de los 6 sistemas de costos contemplados en el presente artículo. El segundo grupo está constituido por 24 preguntas en total, a razón de 4 preguntas por cada uno de los 6 sistemas de costos.

En el primer grupo, los sistemas de costos no se presentan de manera explícita, con la finalidad de corroborar las respuestas dadas en las siguientes 24 preguntas, con el propósito de determinar qué sistema o sistemas de costos se emplean en cada una de las PYMEs. En el segundo grupo se presentan de manera explícita los 6 sistemas de costos, constando cada uno de ellos de 4 preguntas en los que se han considerado 4 criterios clave para identificar el sistema de costos que las PYMEs emplean, sean conscientes de ello o no.

De acuerdo a los resultados de la Figura 2, los 2 sistemas de costos más empleados por las PYMEs agroindustriales son el costeo estándar y el costo por órdenes. Los 2 sistemas de costos menos empleados son el costo estimado y el costo por procesos. Asimismo, se puede observar que las PYMEs no emplean un único sistema de costos, sino que emplean varios, según las circunstancias o necesidades.

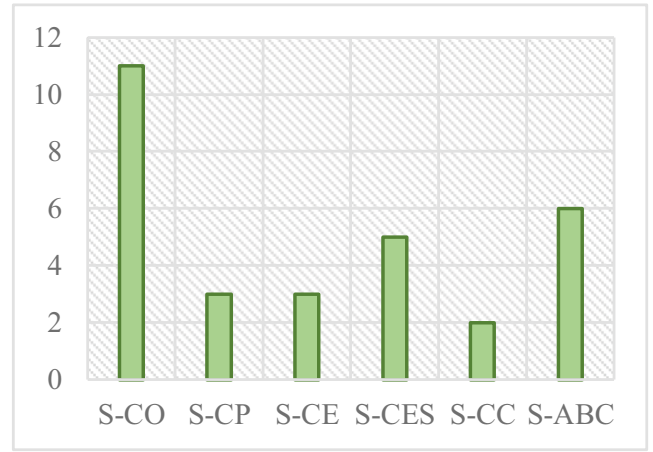

Figura 2. Sistemas de costos que se manifiesta emplear

Fuente: Elaborado en base a la encuesta

Como se puede observar, se confirma lo manifestado previamente sobre los sistemas de costos, que las PYMEs manifiestan emplear más. En algunos casos, incluso, las PYMEs emplean los 6 sistemas de costos.

Según los resultados de la Figura 3, el sistema de costos que más emplean las PYMEs agroindustriales de la Región Tacna es el costeo por órdenes. En menor medida, también emplean el costeo ABC y los costos estándares. Como se aprecia, esto no coincide del todo con los resultados de la Figura 1, en el que los 2 sistemas de costos claramente más empleados, según lo que manifestaban los encuestados fueron: costos estándar, y luego, costos por órdenes. 


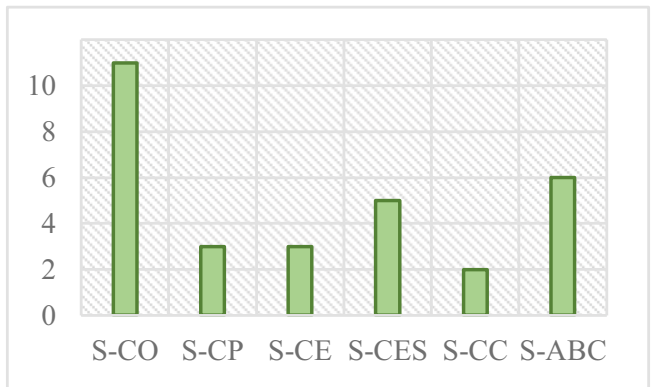

Figura 3. Sistemas de costos que realmente emplea.

Fuente: Elaborado en base a la encuesta

Haciendo un análisis cruzado de los resultados, se tiene que, solo una empresa que manifestó emplear un sistema de costos, realmente empleaba ese mismo sistema de costos. Otra empresa que manifestaba emplear dos sistemas de costos, realmente empleaba uno de ellos. En el caso de otras 9 PYMEs que manifestaban emplear más de tres sistemas de costo, realmente empleaban uno de ellos (8 PYMEs) o dos de ellos (1 empresa). En el caso de las restantes 10 PYMEs que manifiestan emplear algún sistema de costos, no lo emplean en realidad.

Por otro lado, de acuerdo a los resultados obtenidos de la Tabla 4, se verifica con la prueba estadística T de Student a un nivel de confianza del $95 \%$, que la efectividad del control de costos en las PYMEs agroindustriales de la Región Tacna es alta.

Tabla 4

Nivel de control de costos en las Pymes agroindustriales de la Región Tacna

\begin{tabular}{|ccc|} 
& Frecuencia & Porcentaje \\
\hline Bajo & 1 & $5 \%$ \\
Regular & 3 & $14 \%$ \\
Alto & 17 & $81 \%$ \\
Total & 21 & $100 \%$ \\
\hline
\end{tabular}

Fuente: Elaborado en base a la encuesta

De este modo se demuestra en la Tabla 5, donde se verifica con prueba estadística T de Student y un nivel de confianza del $95 \%$, que la efectividad empresarial en las PYMEs agroindustriales de la Región Tacna es alta.
Tabla 5

Efectividad empresarial en las Pymes agroindustriales de la Región de Tacna

\begin{tabular}{|ccc|}
\hline & Frecuencia & Porcentaje \\
\hline Bajo & 0 & $0 \%$ \\
\hline Regular & 2 & $10 \%$ \\
Alto & 19 & $90 \%$ \\
Total & 21 & $100 \%$ \\
\hline
\end{tabular}

Los resultados de la presente investigación se sustentan en la encuesta y el trabajo de campo. Por lo tanto, se puede afirmar que existe una tendencia por parte de los encuestados a reconocer la importancia de la gestión de costos para garantizar la toma de decisiones que permitan el logro de las metas propuestas, lo que trae como consecuencia la necesidad de saber cómo planificar, organizar, ejecutar y controlar para mejorar su estructura de costos y tomar decisiones acordes con sus necesidades actuales.

\section{CONCLUSIONES}

La efectividad de la gestión de costos en las PYMEs agroindustriales de la Región Tacna, 2015 es alto. Se evidencia en las PYMEs agroindustriales de la Región de Tacna que al cumplir con los objetivos planificados, organizados, ejecutados y controlados se tiene en la producción los resultados deseados en los productos agroindustriales.

En las PYMEs agroindustriales se evidencia una adecuada planificación de costos. Sin embargo, se manifiesta en una proporción menor, pero significativa, que no se emplea modelos para medir las unidades dañadas y reprocesadas, ello no permite tomar decisiones para manejar los costos en busca de su disminución y prevención.

Una adecuada planificación no es suficiente para la efectividad de la gestión de costos en una empresa. Los resultados obtenidos en la encuesta aplicada en la presente investigación 
evidencian una buena organización y ejecución de las PYMEs agroindustriales; sin embargo, las PYMEs no aplican las Normas Internacionales de Información Financiera para asegurar la calidad de la información contable, para que se alcance los objetivos planteados.

Las PYMEs agroindustriales de la Región Tacna no emplean un único sistema de costos, sino que emplean varios según las circunstancias o necesidades. El no tener definido un buen sistema de costos ocasiona que no se tenga un adecuado conocimiento y esto no permite emplear información fehaciente sobre los costos para tomar mejores decisiones. Por lo tanto, existen problemas con la implementación de la mejora continua, ya que no se puede gestionar adecuadamente lo que no se puede medir, debido a que se han detectado incongruencias entre el sistema de costos que se indica emplear y el que realmente se emplea.

El control es la última fase primordial para las PYMEs, ya que permite verificar cual es la situación real, como mecanismo que informe si los hechos van de acuerdo con la ejecución de lo planificado. Se evidencia un grado de control adecuado; sin embargo, se refleja que las diversas áreas involucradas en control de costos no conocen la información, indicadores y metas que posibiliten el cumplimiento conforme a lo establecido.

Se evidencia una aceptable efectividad en las PYMEs agroindustriales al cumplir con los objetivos planificados, organizados, ejecutados y controlados, pero los procesos empleados no cumplen con la planificación realizada siendo una desventaja para el entorno competitivo en que se desenvuelven.

\section{RECOMENDACIONES}

Las PYMEs agroindustriales deben implementar un modelo de gestión que responda a una adecuada planificación, organización, ejecución y control, generando la efectividad en las PYMEs. Los productos agroindustriales son muy cotizados en el exterior; por lo tanto, deben ser competitivos, cada vez mejor en calidad y en costos para así lograr el desarrollo económico de la Región Tacna.

Se recomienda planificar las inspecciones intermedias y finales en la misma línea de producción para tener en claro cuál es la cantidad que la empresa tomará como ocurrencia normal mediante informes diarios con personal calificado, que servirá para llevar a cabo un adecuado costeo. Para ello, es necesario diagramar el proceso de producción, verificar las unidades físicas, calcular las unidades equivalentes, los costos unitarios y así agregar al costo las unidades dañadas normales y cargar al Estado de Resultados las unidades anormales determinadas.

Se debe emplear adecuadamente las Normas Internacionales de Información Financiera en las PYMEs agroindustriales a fin, de tener información razonable, comparable y de máxima calidad que servirá para facilitar la toma de decisiones en la fase de control de costos. Además, es necesario considerarlo como estrategia de medición del desempeño en las PYMEs agroindustriales, lo que permitirá llevar a cabo evaluaciones que garanticen el cumplimiento de los objetivos establecidos. El adecuado manejo de los costos con los estándares internacionales optimizará la efectividad de las PYMEs agroindustriales.

Se debe implementar un modelo de costeo razonable y adecuado para las PYMEs agroindustriales, para evitar tomar decisiones equivocadas y proporcione información confiable, objetiva y oportuna para la dirección. Un buen sistema de costos ayudará a la gerencia a tomar decisiones apropiadas para los costos anticipados de fabricación y precios de ventas, así como las utilidades estimadas. Constituyéndose como un ejercicio gerencial.

Se deben implementar en su totalidad las Tecnologías de la Información y Comunicación, es decir, las TICs con toda la información contable relevante y a disposición 
de los interesados que laboran en las áreas que se encuentran involucradas para que conozcan todo lo concerniente a ella. Es fundamental tener un sistema de información para una adecuada gestión, para tener una mayor eficiencia y calidad en el registro de las operaciones contables al producir información veraz, confiable y oportuna que permitirá que la gerencia esté en la posibilidad de tomar decisiones acertadas.

Las PYMEs agroindustriales deben emplear para sus procesos un enfoque estratégico en el producto, que se caracteriza por producir grandes cantidades para poca variedad de productos. Ello permitirá un adecuado control en los costos, ya que los costos totales dependen mucho de la utilización de la capacidad instalada y facilitará así los procesos de certificación de calidad al ser un flujo continuo y estandarizado.

\section{REFERENCIAS BIBLIOGRÁFICAS}

Banco Central de Reservas del Perú (2013). Informe Económico y Social Región Tacna. R e c u p e r a d o d e http://www.bcrp.gob.pe/docs/Proyecc ion-Institucional/EncuentrosRegionales/2013/tacna/ies-tacna2013.pdf

Chaparro, M. y Piragua, S. (2015). Modelo de Gestión Estratégica de costos para la empresa fertilizantes Bocayá Ltda. (monografía para título). Universidad Pedagógica y Tecnológica de Colombia. Sogamoso, Colombia.

Cervera, F. (2011). La Percepción de la efectividad organizacional. (tesis de doctorado). Universidad Autónoma de Querétaro. Querétaro, México.
Cuervo, J. y Osorio J. (2011). Costeo basado en actividades-ABC Bogotá:Eoe Ediciones

Dirección Regional de Agricultura. (2015). Exportaciones de principales productos agricolas de Tacna Periodo: enero-diciembre 2015. Recuperado de http://www.agritacna .gob.pe/gestores/estadistica/of_ol_est

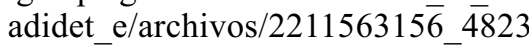
066650.pdf

Dirección Regional de Producción (2016). Padrón MYPE. Tacna, Perú.

Guerra, G. (1992). Manual de Administración de Empresas Agropecuarias. San José, Costa Rica: IICA

Hernández, E. (2007). Contabilidad financiera y gestión de costos. Santa Clara, Cuba.

Hervas, M. y Ponce, I. (2007). Diseño del Sistema de Gestión de Costos de la Empresa Ediecuatorial (tesis de pregrado). Universidad San Francisco de Quito, Ecuador.

Mejía, N. (2013). Sistema de Indicadores Responsables. Maracaibo, Venezuela.

Mendieta, B. (1996). Administración Agropecuaria. Managua, Nicaragua

Ministerio de la producción. (2013). Decreto supremo $N^{\circ}$ 013-2013-Produce.

PMBOK Project Management Body of Knowledge (2013). Guía de los fundamentos para la Dirección de Proyectos. EE.UU.: Project Management Institute, Inc

Rincón, C. y Villareal, F. (2010). Costos Decisiones empresariales. Bogotá, Colombia: Ecoe Ediciones

Udolkin, S. (2015). Contabilidad de costos y de gestión. Lima, Perú: Universidad del Pacifico. 